\begin{tabular}{ccc}
\hline & International Journal of Engineering \& Technology, $7(2.7)(2018) 230-236$ \\
SPC & International Journal of Engineering \& Technology \\
Website: $w w w . s c i e n c e p u b c o . c o m / i n d e x . p h p / I J E T$ & Research Paper \\
\hline
\end{tabular}

\title{
detection of indulgence attack adopting multivariate correlation analysis
}

\author{
P. Divya ${ }^{1 *}$, M. Saikiran ${ }^{1}$, K. Nagarjuna ${ }^{1}$, K. V. D. Kiran ${ }^{2}$, Ch. V. Phani Krishna ${ }^{2}$ \\ ${ }^{1}$ IV/IV BTECH Students Department of Computer Science And Engineering K L E F Deemed to be University, Vaddeswaram, \\ Guntur Dt, Andhra Pradesh, India \\ ${ }^{2}$ Professors Department of Computer Science And Engineering K L E F Deemed to be University, Vaddeswaram, \\ Guntur Dt, Andhra Pradesh, India \\ *Corresponding author E-mail: divyaputtagunta21@gmail.com
}

\begin{abstract}
Different types of the servers such as Net, data, distributed computing and then forth, has been mutually connected. In current days all these servers are covered twist from the arranged aggressors. More often than of universal \& commanding esteems, "Denial-of-Service" (DoS) offensive purpose honest tremble on figuring schemes. Here, I can display a framework of the DoS assault location which uses the Multivariate Correlation Analysis that is shortly called as MCA as long as the system movement factual replica of through dividing relationships based on geometric within system activity main features. Implemented "DoS assault identification framework based on MCA" uses a irregular condition situated place in the acknowledgment of the assault. it accomplishes settlement fit to catch insoluble \& certified "DoS assaults" modestly with the help of the research samples of the "true blue system activity".
\end{abstract}

Keywords: Service Attack Denial; Multivariate Correlations; Network Traffic Characterization; Area of the Triangle Area.

\section{Introduction}

A threatening meddling sort of conducting persuasive and for online servers is the DENIAL-OF- Administration assaults. The aggression of the DoS really weakens Casualty receptiveness, that may be an switch; hub; a host; / a entire system. They bring out serious expected assignment for casualty through the flood of it including the amazing pointless parcels measurement; or its method weakness corrupting. From minor says to the alike set of minutes, a casualty might be embarrassed in connection with administration It causes the factual harms for the administrations that are running on casualty. Therefore; DoS viable finding assaults are the essential for the online administrations security. Working on the DoS assault location is one the crucial examines for the system of the discovery strategies-based improvement. Location Methods in light; the strategies screen activity of transmitting on the safe guarded systems. The shielded online servers are discharged by these services from; checking assaults; It gives the guarantee to servers that can be devoted themselves for furnishing the administrations quality based on a least deviation accordingly. "Dispersed crafty booking" (DOS) is simply higher than planning of the entrepreneurial customary. since, the focal substance which is the nonappearance gives all stations channel conditions. [6]. The frameworks that are Interconnected, for illustration, servers, of the distributed computing; database server; and web servers; and so forth., are currently under strings from arrange aggressors. Here, Basic assault is "Denial of Service"; which assaults origin genuine effect s registering methods [8]. Among all the today's internet solid security issues; the "Disavowal of Service" (DOS) assaults are danger boundless for the web locales. So, the its is not an easy thing to find in Denial of service internet. DoS assaults problem will be removed is an outstanding. "Conveyed Denial of Service" (DDoS) assault may be the Scale of the boundless, forward assault upon the casualty framework of administrations accessibility; asset of the system, drive in the round about track with the help of number of Personal Computer's traded off on Internet. every growing DDos problem answers are the thought of the experts. [10]. By using the DOS, irregular access can be done by stations [9] uses for debating a channel \& after opposition winning, channel states also measured. In the wake of measuring a channel conditions this submitting the opportunity of the transmission when quality of the channel is not good.; Often, when the quality of the channel has been excellent then the station can be transmitted. The DOS Circulated Idea can make the clients who are narrow minded is defenseless. that type of the client selects more Offer in the prominent remote assets at all-round carried on the client's charges with the help of greater open doors of the transmission; and the deviating from.

\section{Overview of dos attacks}

\subsection{Denial of service type}

"Denial of Service assault" have specified with the raiders for counteracting authorized administration clients through an open and shut effort from using in order that administration. Illustrations organizes, enterprise to amazed clubs in amid 2 devices forward available lines to skip the access for administration, enterprisers for surging a method or a system, along this track "forestalling true blue system traffic". Cracks for keeping a particular individual to the administration, Attempting the for administration disturbing to specific framework;/ individual. To keep up the Specifications Integrity [10] The given figure describes relevant "Denial of Service Attack" structure. There are 3 segments like attackers; an internet $\&$ object 
in DoS structure where an assailant can be assaulted for user version from their service access.

DoS Attacks

A massive number of nasty packets sent by one machine for the use of targets network resources \& computational; or crashing; the target exhaust. The main motto of these type of violations is for wasting a specific user access to the services of the target's.

An internet connection with a computer can be utilized for flooding the server with packets in A DOS violation. for the purpose of overloading the target devices band widths and resources. [10]. various DoS classification of the Attacks can be given in the following sections.

- Network Device Level:

Network Device Level attacked by DOS. Which consists of attacks that may occur with either bugs of the software or for exhausting network devices hardware resources [10].

- Operating System Level:

At DOS i.e OS Level attacks taking an advantage on operating systems; implement protocols ways [10].

- Application based attacks:

A machine or; the service may go to out of order; or occurs various bugs at specified host or device when huge number attacks occurs. Data Flooding:

Based on the having Bandwidth; network; host; device; the attacker sends a heavy amount of the data. As a result, terribly, broad amounts of data can be processed [10].

- Protocol features of the Attacks:

Certain standard protocol features are the features of the DOS.

\section{Proposed work}

Implemented "DoS attack detection system architecture" overview explained; along with scheme framework; \& mechanism observation. There, 3 levels in entire detection process.

Level 1: Multivariate correlation analysis

Level 2: Generation of the Profile as Normal.

Level 3: Detection of the Attack.

\subsection{Proposed architecture}

Level 1:

Basic features from the the network traffic can be generated in 1st level. that give access to a network which is placed internally called as internal network. In this network; the servers reside which are proposed that are useful for forming the records of the network traffic in a robust- specified time schedule. In the suitable traffic of inbound, we can decrease the envious moments through observing; and analyzing. For offering the best safeguard to target the internal network. The detector can be activated by this internal network for offering the protection. This can be well suitable particular internal network. As the number of smaller network services is used in detectors, legitimate traffic profiles are useful to it.

Level 2:

Coming to the 2nd step, the "Multivariate Correlation Analysis" has been given to the module of the Area of the Triangle Which Generates a Map. It will be given to separate a correlation of the 2 different features of the; individual traffic record. in this track, Specific features are out from the 1st level or "feature normalization module". Entire correlation that has been extracted will be stored in one space which is known as "Triangle area Map" (TAM); It will be helpful for replacing original records.

\section{Performance resolution}

- Detection Rate:

It can be defined as the count of intrusion instances observed with the help of a true positive system will be divided with total amount of presented test set intrusion instances.

Rate of False Alarm:
Count of usual patterns restricted like attacks which is known as False positive can be separate with a complete count of the typical patterns.

Simulation of The Alerts:

True Positive: Attack; - Alert

True Negative: No attack; - No Alert

False Negative: Attack; - No Alert

False Positive: No attack; - Alert

Terms:

True Positive: For producing the alarm, it is a legitimate attack that activates IDS.

False Positive: If there is no attack then the alarm will be given by the event signaling IDS.

False Negative: If any attack occurs then there is no alarm will be raised.

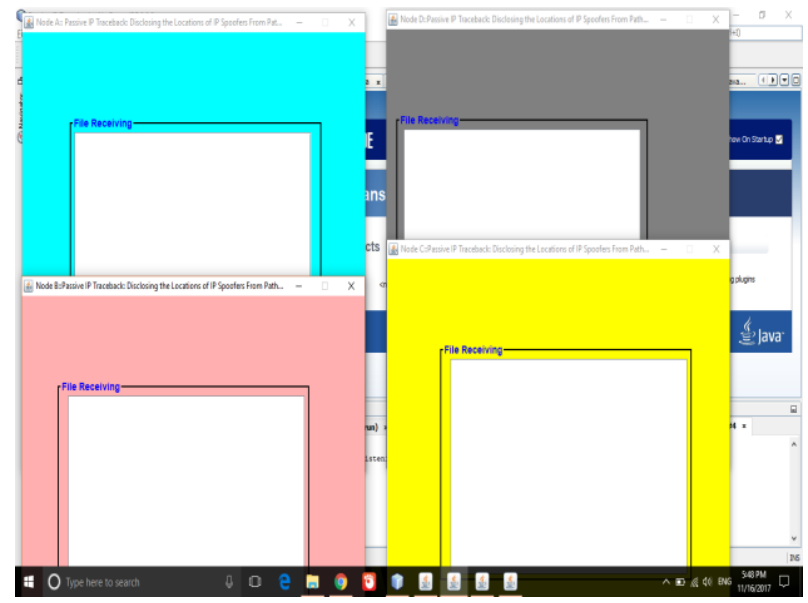

Fig. 1: These are the Receiving Windows Where the Data Will Be Displayed after Receiving the Data from the Sender

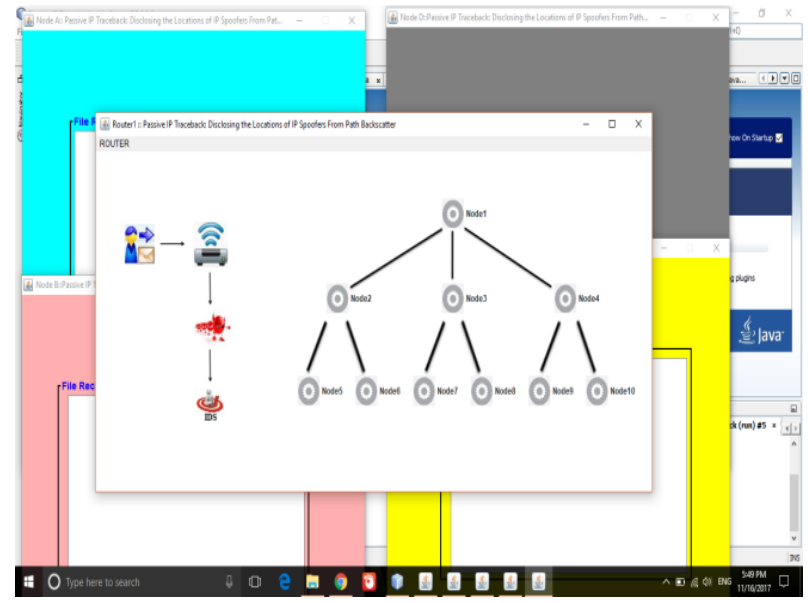

Fig. 2: At this Point the Router Becomes Active and the Corresponding Elements are the Used Once where they Are Sender, Router, IDS.

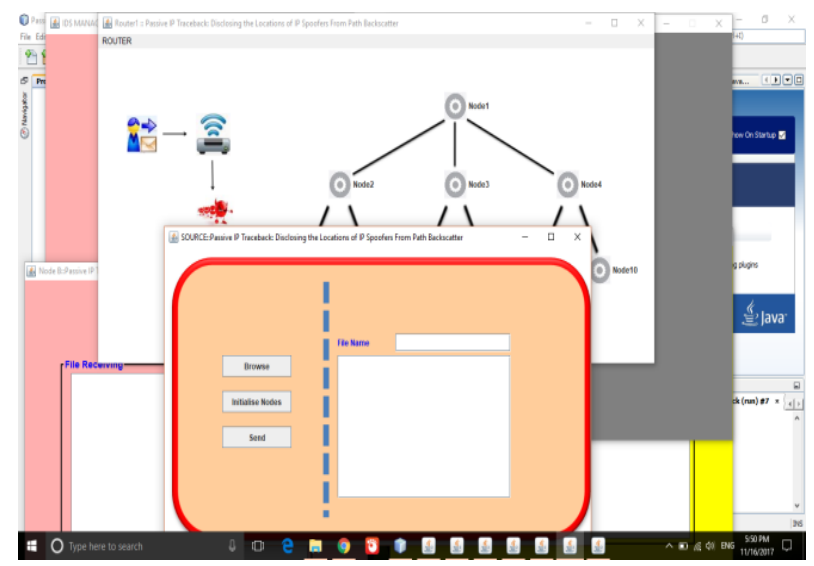

Fig. 3: Here we are Able to Browse Our Files and Also, We Can Write A Message in the Text Box Displayed. 


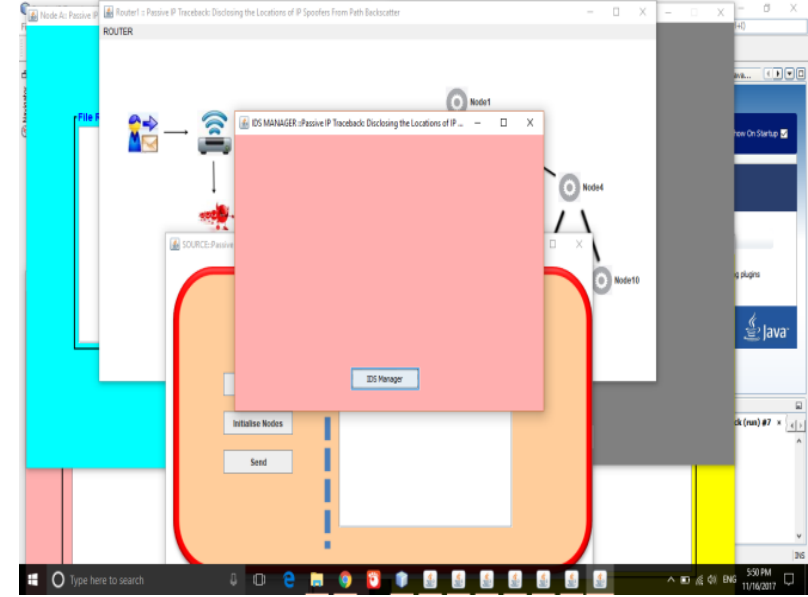

Fig. 4: IDS Manager Is the Module Used for Displaying the Data to Which Node and with what IP Address the Data Has Been Sent.

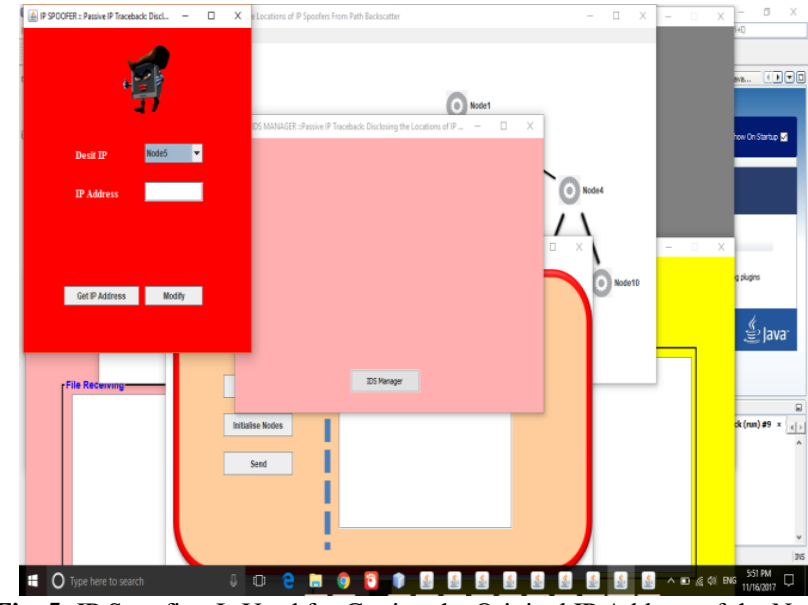

Fig. 5: IP Spoofing Is Used for Getting the Original IP Address of the Node Selected and Modify the Original IP Address of that Specific Node by Using the Spoofing Technique Shown.

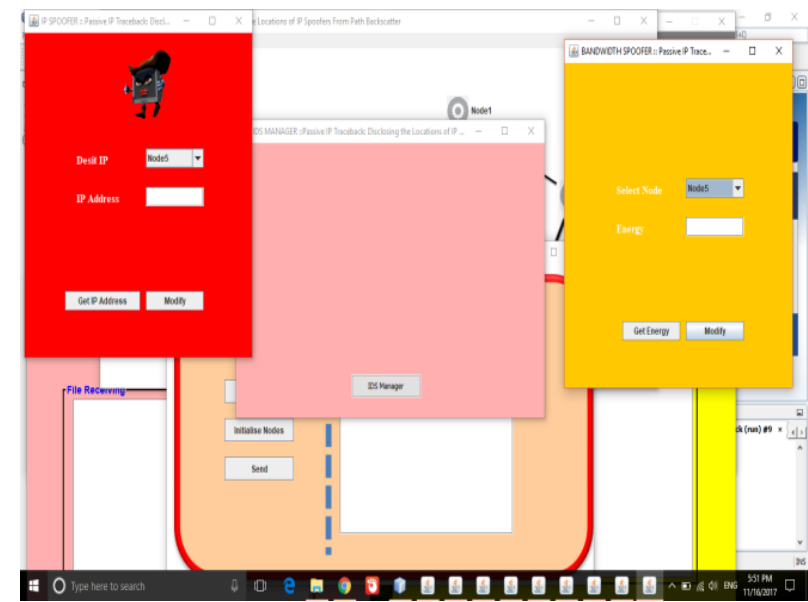

Fig. 6: Bandwidth Spoofing Is that Every Node Has A Specific Bandwidth and If that Bandwidth Is Spoofed the Process Gets Under Attack and the Data Is Going to be Spoofed.

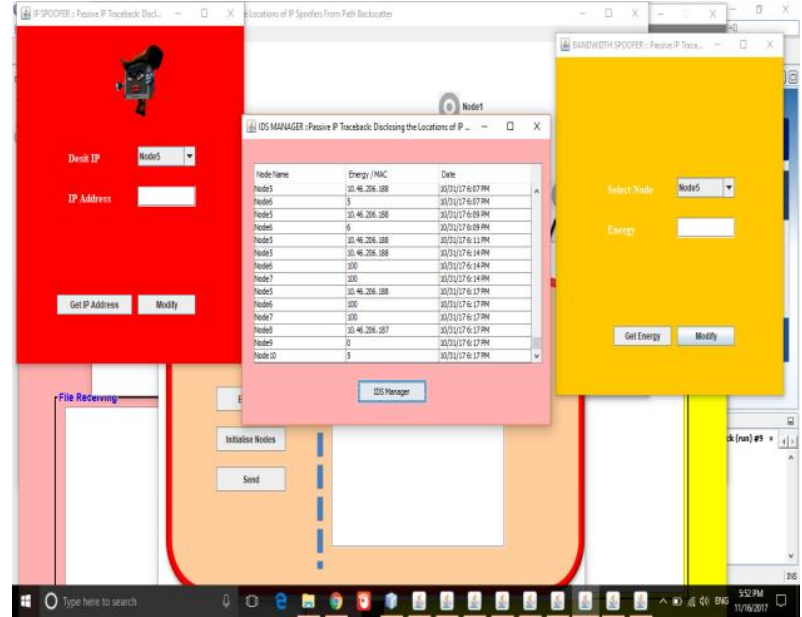

Fig. 7: IDS Manager Is that Displays All the Transfers that are Done in the Project where It Specifies the IP Address, Node Name, Date, and Time As Displayed in the IDS Manager.

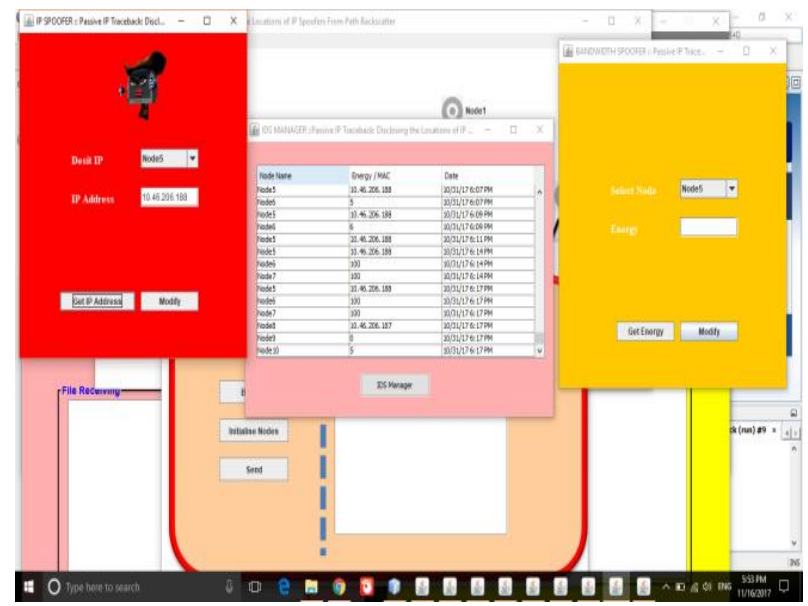

Fig. 8: At this Step, We Have Selected the Node 5 and We are Able to Get the Original IP of the Node5 in the Router Where It Can Be Proved from the IDS Manager Displayed on the Screen.

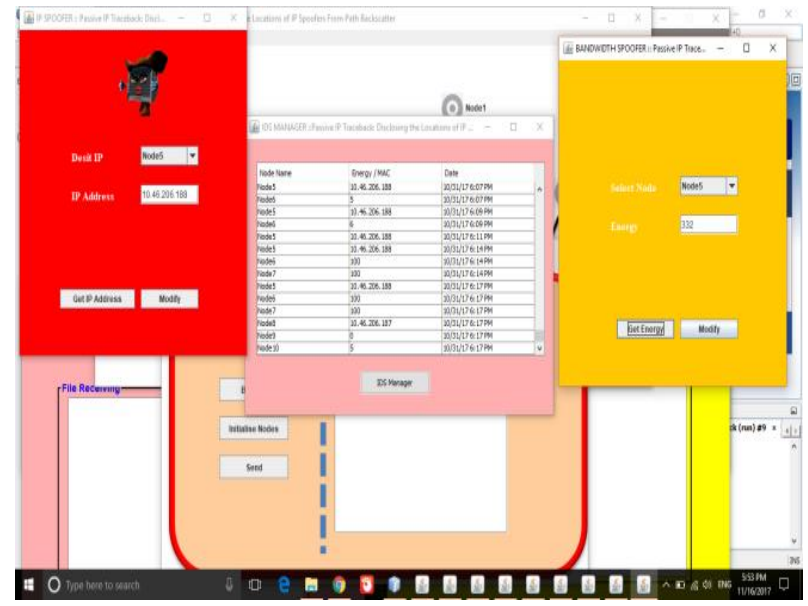

Fig. 9: Here the Bandwidth of A Specific Node Is Displayed in the Energy Box Where It Is the Original Bandwidth and It Can Be Modified by Using the Option Modify As Given in the Module of the Bandwidth Spoofing. 


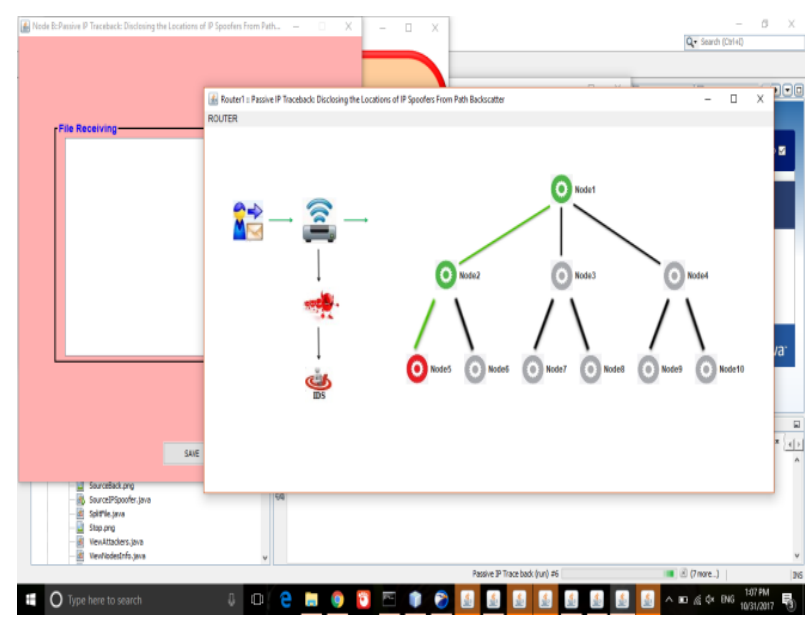

Fig. 10: Here the Process Is Started and Inside the Checking Is Going on by Checking Every Node that Is Possible to Send the Data and at Which Node the Model Could Drop the Data.

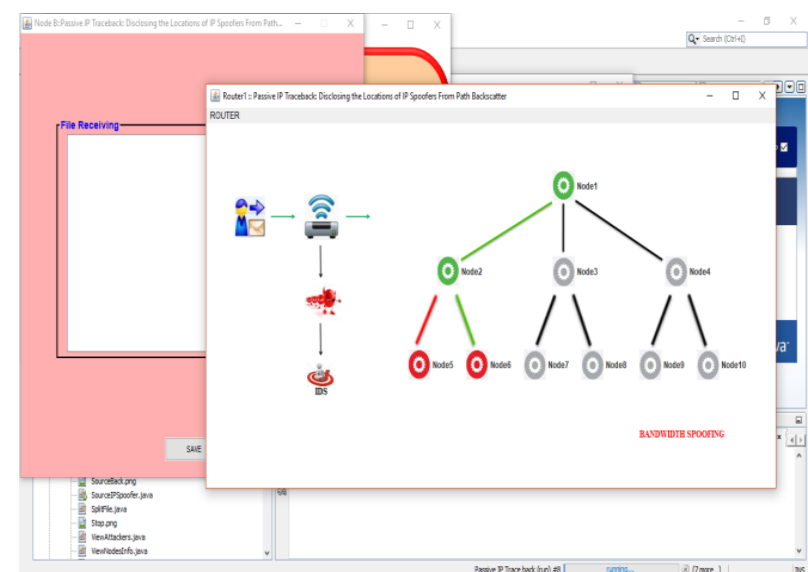

40

Fig. 11: Here the Node5 Is Rejected and Backtracked to the Previous Nod and Checks the Node6 and where It Is Also Rejected As Shown. and the Reason Why the Node 5 and Node6 Are Rejected Is that those Nodes R Not Capable of Sending the Data where There May Be A Defect in the Bandwidth Attained to those Nodes.

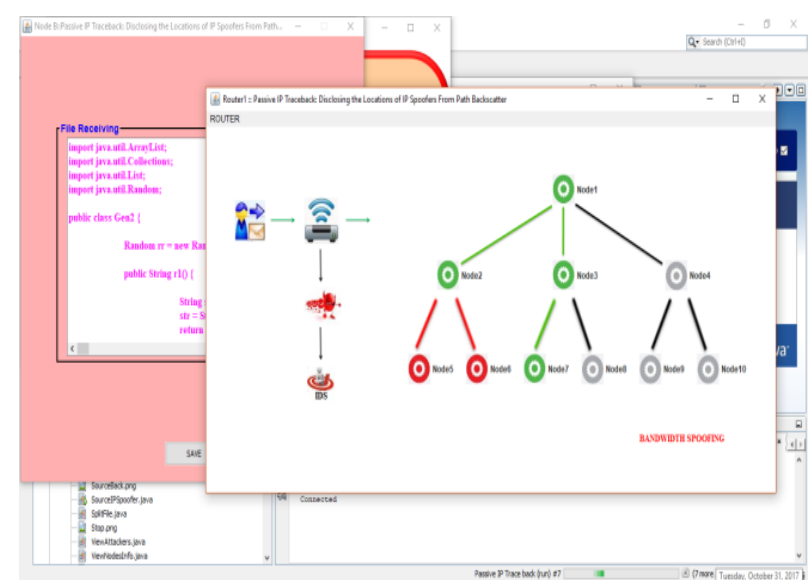

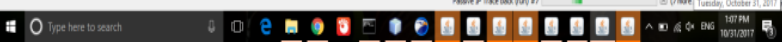

Fig. 12: At this Point after Backtracking the Node at Node 7 the Data Can Be Able to Be Sent and then the Data Is Sent Through the Node7.

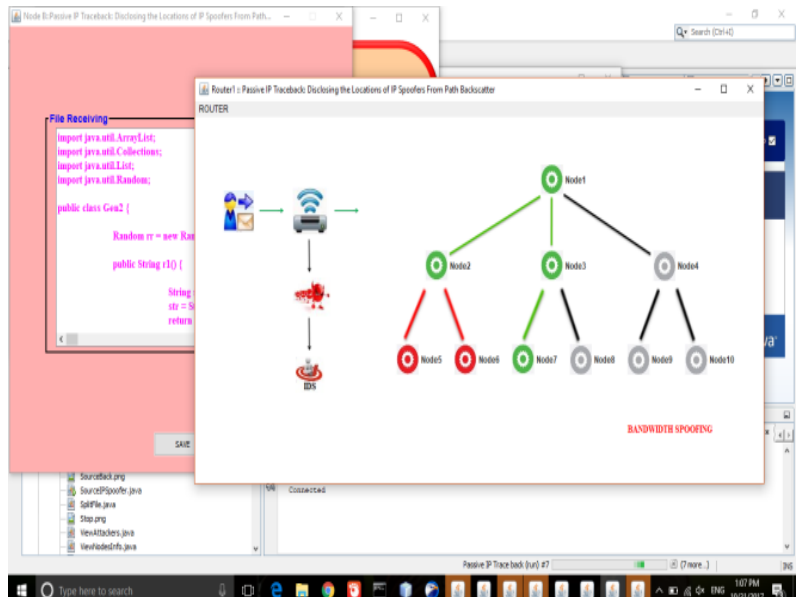

Fig. 13: As If We Observe the Background the Data Is Received in the Node B which Is Selected in the Starting to which Node the Data Is to Be Sent.

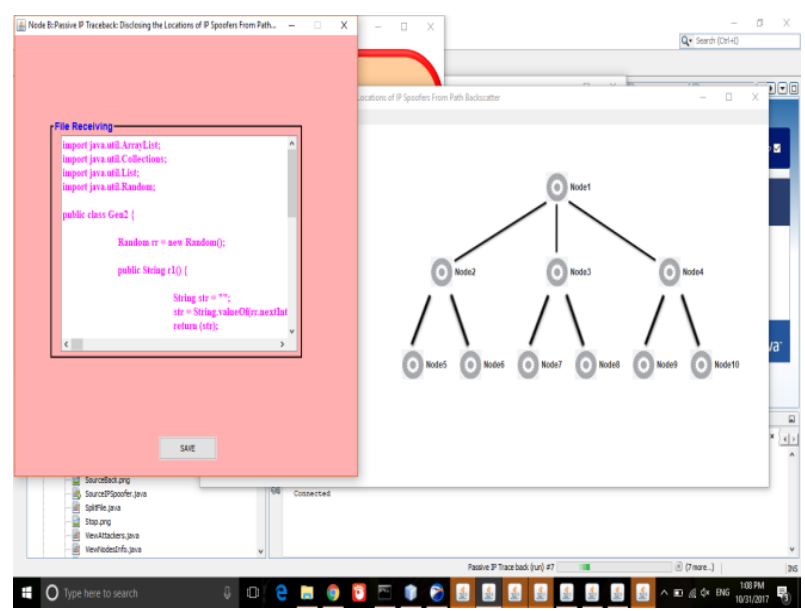

Fig. 14: Here Is the Data That Sent from the User to the Node B As We Have Given at the Starting of the Process.

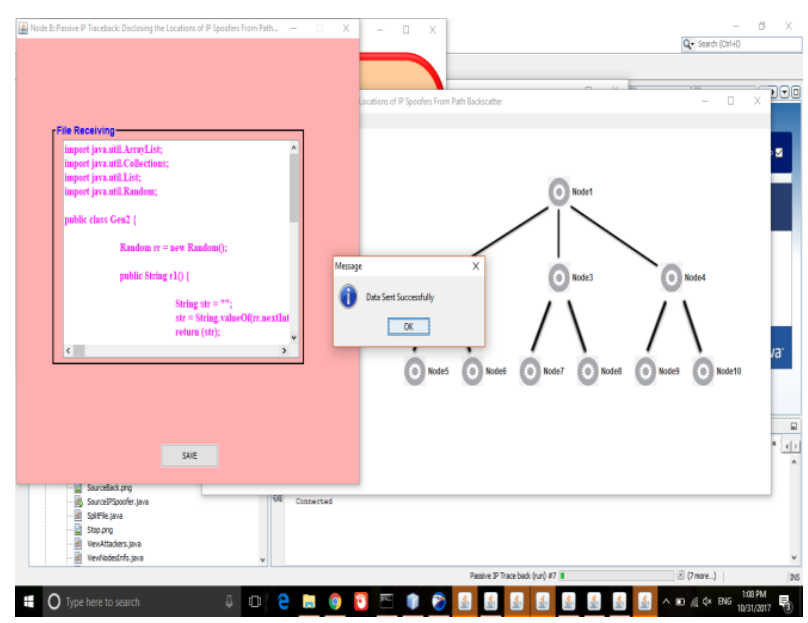

Fig. 15: This Is the Dialogue Box Displayed after Delivering the Data to the Corresponding Node that Is Given. 


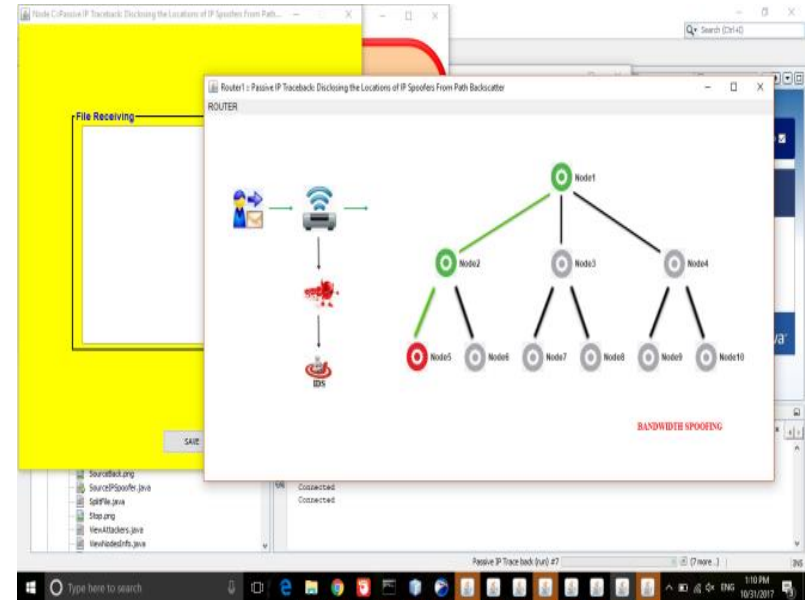

Fig. 16: At This Point the Process Is Started and the Nodes R Being Tested for Sending the Data in A Sequence.

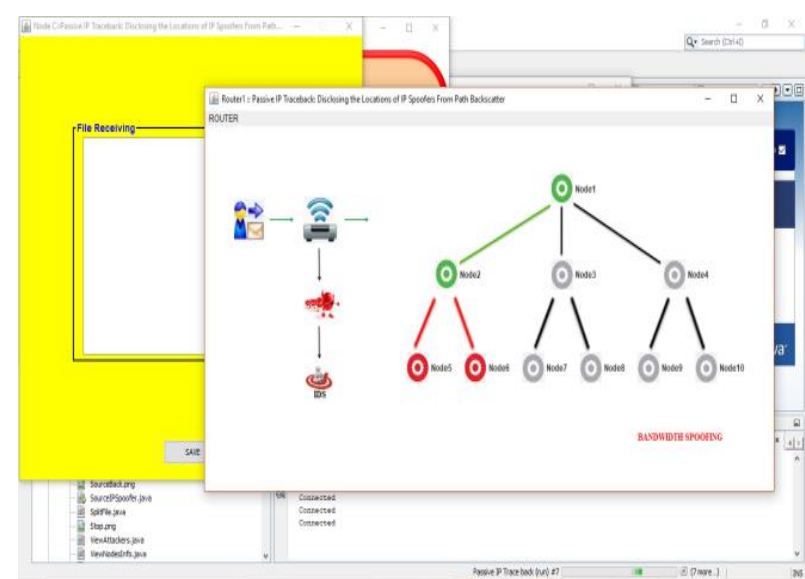

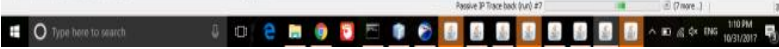
Fig. 17:

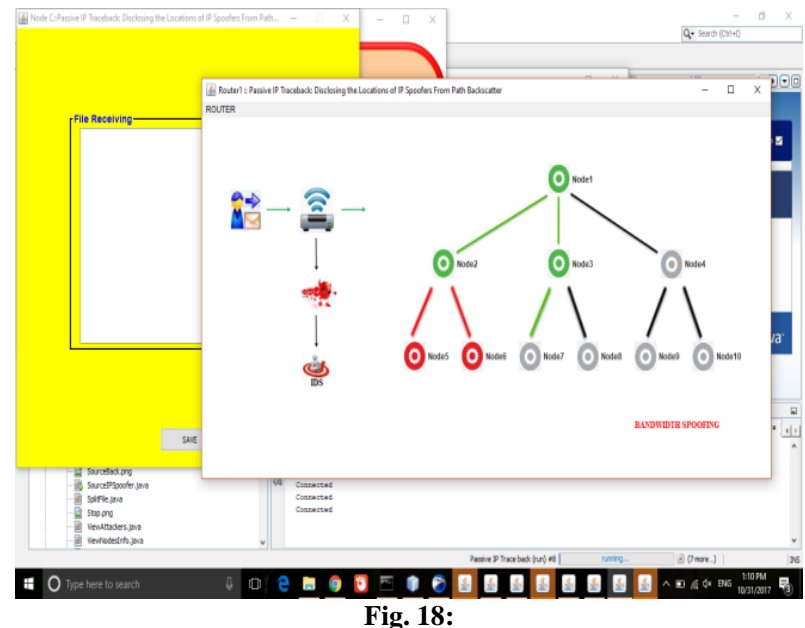

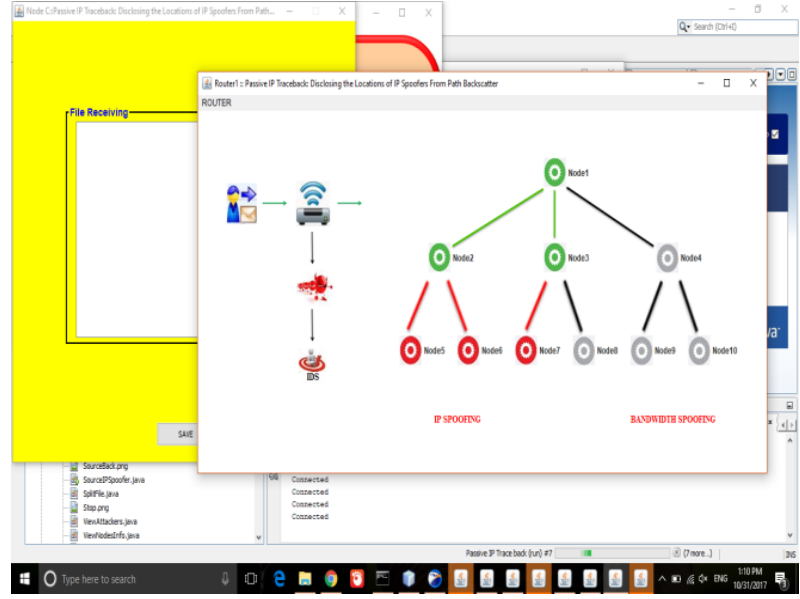

Fig. 19:

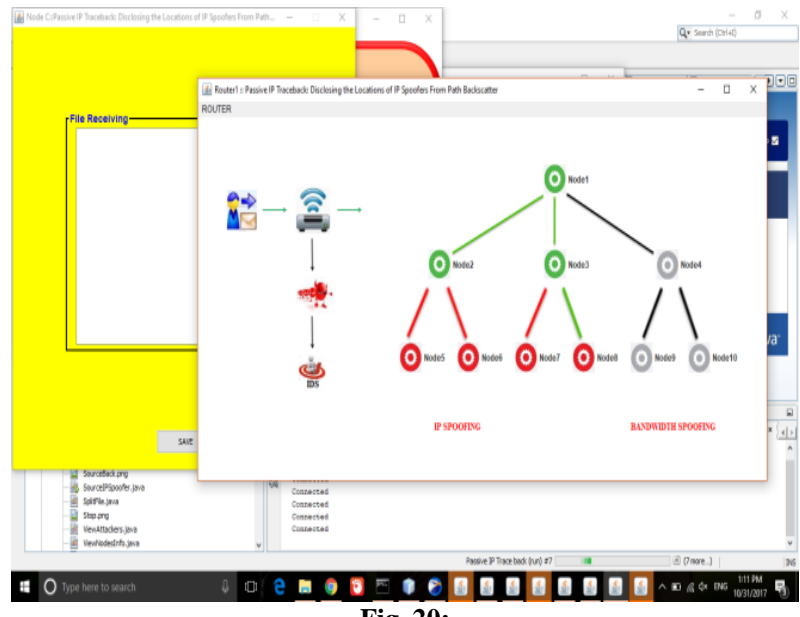

Fig. 20:

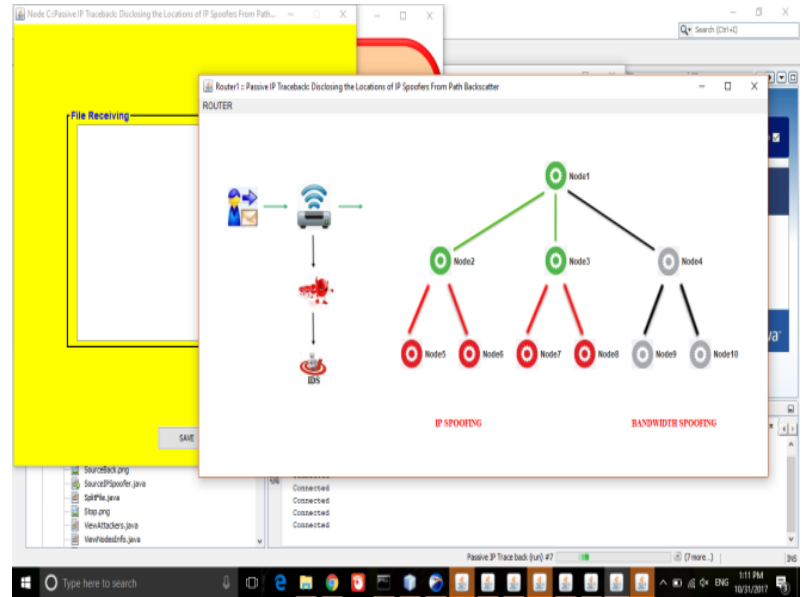

Fig. 21: This Is the Process of the Router Checking the Possibility of Sending the Data Through Which Node that the System Is Able to Send the Data to the Receiver. 


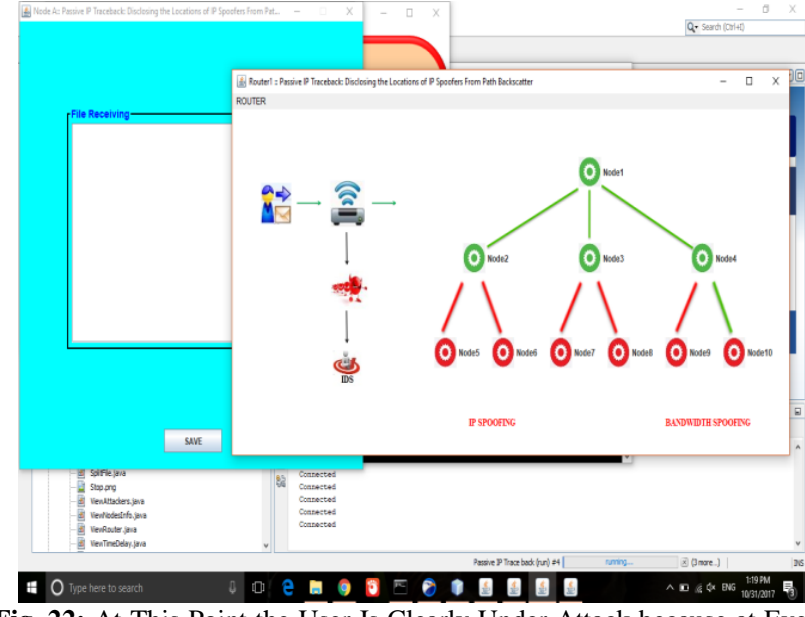

Fig. 22: At This Point the User Is Clearly Under Attack because at Every Node the Data Is Not Received and Says that the Service Is Under Attack, where the IP Address or the Bandwidth are Spoofed by A Fake One.

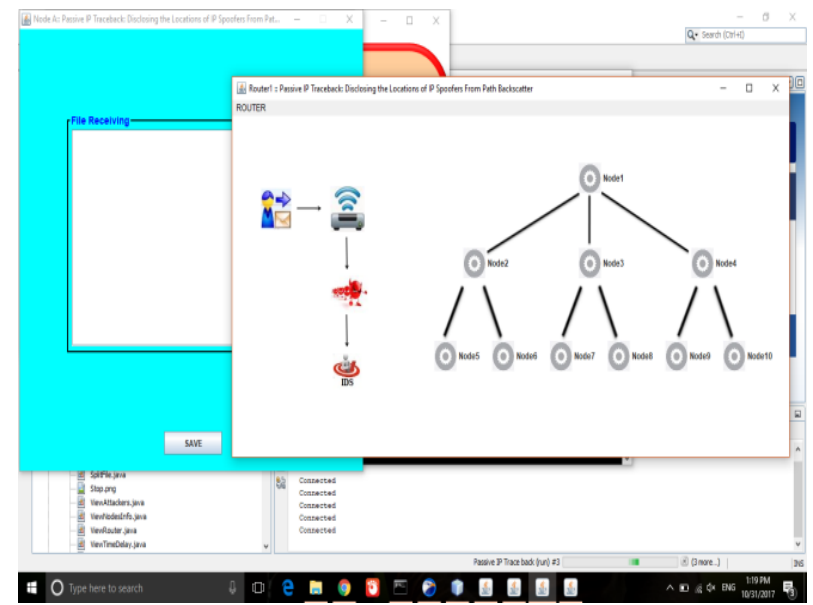

Fig. 23: As A Proof No Data Is Received at the Receiving Node As We are Seeing the Node Box Is Empty and Also Says No Data Received after the Process.

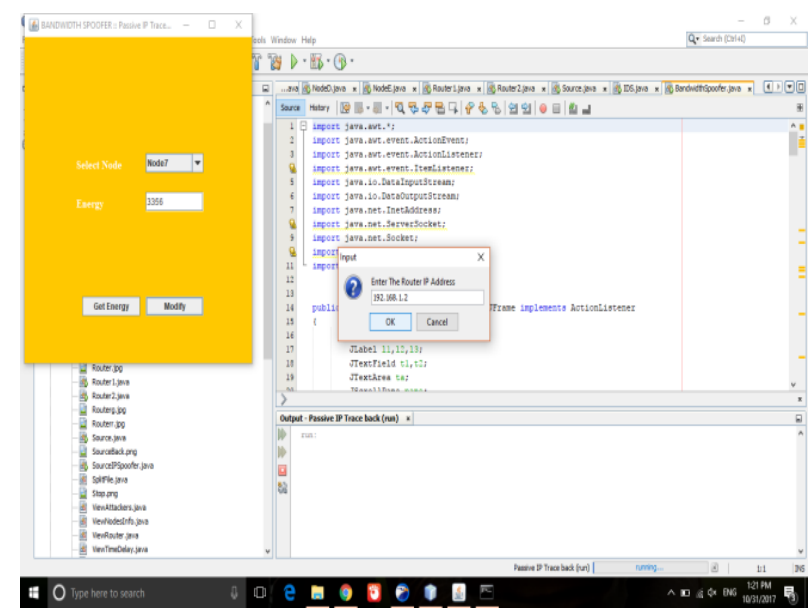

Fig. 24: Here the Bandwidth of the Node 7 Is Getting Modified As the System Can Be Attacked Through Changing the Bandwidth of the Node and Submitting the Original IP Address So that It Can Be Changed Without Any Error.

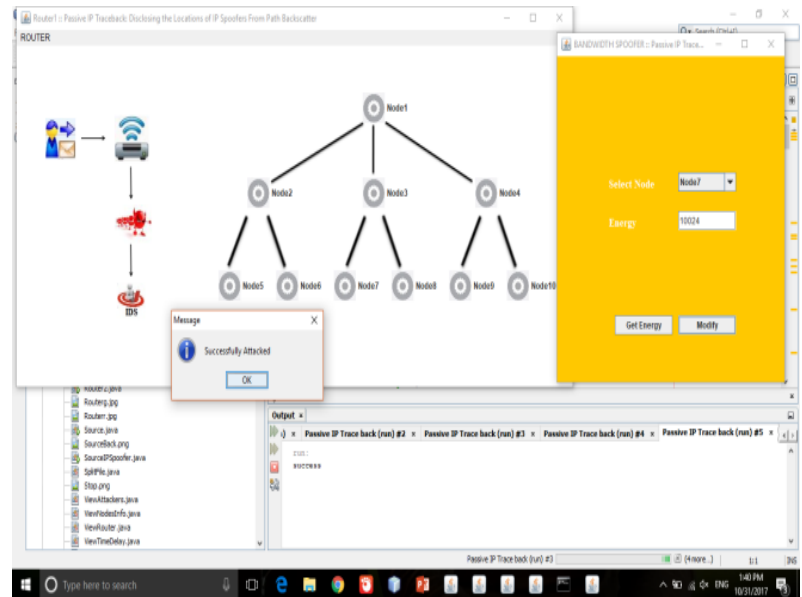

Fig. 25: After the Change of Bandwidth of the Node As A Result the Message Box Shows the Message that the Attack Is Successfully Done.

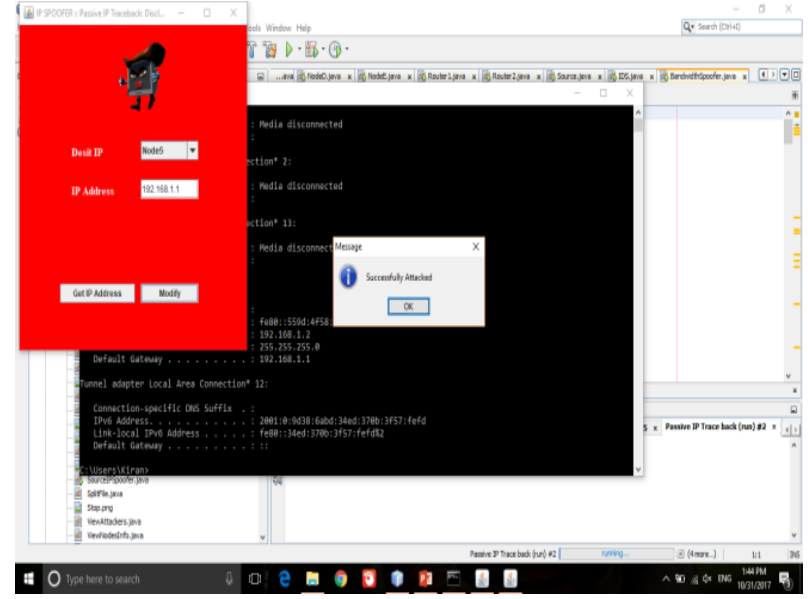

Fig. 26: Not Only by the Bandwidth Spoofing We Can Also Attack Through the IP Spoofing, Here the Original IP Address of the System Is 192.168.1.2 As Displayed in the CMD in the Background and We Have Used IP Spoofing Technique and Modified the IP Address As192.168.1.1 and the Result Is Successfully Attacked As Shown in the Message Window.

\section{Conclusion}

The implemented "MCA-based DoS attack detection system" has been developed with the help of two main techniques. Those are MCA technique based on triangle area \& detection technique situated by anomaly method. In every traffic record of the network, Ancient methods are mainly concentrating on hidden correlations of the geometric 2 different features. In traffic behaviors network, those are also gives Systematic characterization. Our system i.e latter technique helps in separating the unknown DOS and known DOS which are drives from suited network traffic. The Time complexity can be decreased, and on real time dataset, the Results has been taken. The implemented method deduced the false positive rate also.

\section{Acknowledgement}

This work is supported by the Department of Science and Technology, India through the fund sanctioned for improvement of Science \& Technology infrastructure, at department of CSE, K.L University, by order number SR/FST/ESI-332/2013. 


\section{References}

[1] Zhiyuan Tan; Senior Member; Priyadarsi Nanda; Aruna Jamdagni; Xiangjian He; and Ren Ping Liu; "A System for Denial of Service Attack Detection Based on Multivariate Correlation Analysis", IEEE TRANSACTIONS ON PARALLEL AND DISTRIBUTED SYSTEMS; 2013.

[2] J. Haggerty; Qi Shi; "Early Detection and Prevention of Denial of Service Attacks: "A Novel Mechanism with Propagated TracedBack Attack Blocking” IEEE Transaction on, Vol. 23; 2005.

[3] R Nagadevi; P Nageswara Rao; Rameswara Anand; ack "Using Multivariate Correlation Analysis", International Journal of Computational Engineering Research (IJCER, Vol.04; 2014.

[4] G. Thatte; U. Mitra; and J. Heidemann; "Parametric Methods for Anomaly Detection in Aggregate Traffic," Networking; IEEE/ACM Transactions on; vol. 19; no. 2, pp. 512-525; 2011.

[5] Darshan Lal Meena Dr. R.S.Jadon; "A Survey on Different Solutions to DDoS Attacks"; "International Journal of Advanced Research in Computer Science and Software Engineering;" Vol. 4; 2014.

[6] K.V.D.Kiran "Risk Assessment in Distributed Banking System," International Journal of Applied Engineering Research(IJAER)", ISSN 0973-4562 Volume 9, Number 19 (2014) pp. 6087-6100

[7] K.V.D.Kiran," Analysis and Classification Scheme of Risk Assessment Miniatures placd on Different Criteria for Reducing the Risk", International Journal of Applied Engineering Research"pp.1206912085, ISSN 0973-4562 Volume 9, Number 22 (2014).

[8] K.V.D.Kiran,'Information Security risk authority in critical informative systems", CSIBIG 2014 